\title{
LE FANTASTIQUE RÉEL DE FRANZ HELLENS. GENÈSE ET CONCEPTION
}

\author{
Renata BIZEK-TATARA \\ Université Marie Curie-Skłodowska, Lublin
}

\begin{abstract}
En): This article focuses on the genesis and conception of the fantastique réel of Franz Hellens (1881-1972), a Francophone Belgian writer. Before defining its nature, the author goes back to the genesis of the term, to Edmond Picard and his characteristic of Belgian literature, as well as to the Hellens' conception of fantastic as a literary genre. She then studies Hellens' fiction to identify the essence of her fantastique réel which is based on the harmonious reconciliation of the real and unreal, as well as the function of the gaze and dream in the discovery of the supernatural in the real.
\end{abstract}

Keywords (En): Fran $\square \mathrm{H} \square 1 \sqsubset$ ns; fantastique réel; B $\square$ lgian Francophon $\square$ lit $\llbracket$ ratur $\square$; sup $\llbracket$ rnatural; $\mathrm{ga} \amalg ; \mathrm{dr} \sqcap \mathrm{am}$

Mots-clés (Fr): Franz Hellens ; fantastique réel ; littérature belge francophone ; surnaturel ; regard ; rêve

Outre une pléiade de symbolistes, de grammairiens et d'irréguliers du langage, la Belgique francophone a mis au monde une ribambelle de grands maîtres de l'imaginaire. Peu de pays peuvent se vanter d'un nombre si important d'auteurs épris du surnaturel, de vrais orfèvres en la matière, dont chacun, note Jacques DE DECKER, a « une manière bien personnelle de déverrouiller le carcan du réel et d'ouvrir les voies vers l'impossible» (1990: 43). La vertigineuse profusion de textes remarquables et hétérogènes esthétiquement qu'ils fournissent, dans un laps de temps si court ${ }^{1}$, peut même impressionner. En effet, la production non mimétique belge se mue en arabesques nombreuses et fort diverses: elle revêt l'habit du symbolisme mystique de Maurice Maeterlinck et Georges Rodenbach, de la sciencefiction de J. H. Rosny aîné, du fantastique terrifiant de Jean Ray, Thomas Owen et Gérard Prévot, du fantastique crépusculaire de Michel de Ghelderode, du fantastique loufoque de Jean Muno, Maurice Carême et Bernard Quiriny, du fantastique corrosif de Jacques Sternberg et Gaston Compère, du réalisme magique de Paul Willems et Guy Vaes et, enfin, du fantastique réel de Franz Hellens, courant non réaliste qui est le propre de la Belgique, son bien précieux, sa spécificité par laquelle se manifeste la singularité des lettres belges francophones.

Toutefois, bien que le fantastique réel soit l'apanage de la littérature belge et traverse comme un fil rouge toute son histoire ( $C f$. QUAGHEBEUR, 1990 : 95), sa nature ne se laisse pas facilement définir. Cette difficulté tient essentiellement au manque d'une conception claire et achevée de l'esthétique hellensienne, ainsi qu'à la déficience des travaux théoriques qui lui sont consacrés ${ }^{2}$. Toujours est-il que si

\footnotetext{
${ }^{1}$ Rappelons que le fantastique réel, le fantastique et le réalisme magique naissent en Belgique au $\mathrm{XX}^{\mathrm{e}}$ siècle.

${ }^{2}$ Deux ouvrages donnent à ce sujet le plus d'éclaircissements : l'essai sur l'école belge de l'étrange, écrit en polonais par Ryszard Siwek, Od De Costera do Vaesa. Pisarze belgijscy wobec niezwyktości (2001 : 80-93) et $n^{\circ} 21$ de Textyles, publié en 2002 et intitulé «Du fantastique réel au réalisme magique », dirigé par B. Denis avec la collaboration de D. Gravez.
} 
nous comparons la situation du fantastique réel au fantastique, nous constatons facilement que, contrairement à ce dernier, le courant belge n'a guère donné lieu à des investigations aussi pléthoriques et diversifiées. Les articles qui l'abordent se réduisent en général à l'analyse d'une fiction isolée d'Hellens ou à l'étude sociolittéraire de la notion dont il fut promoteur.

Quant à Hellens (1881-1972), il n'avait, à vrai dire, rien d'un théoricien ni d'un fondateur d'école : bien qu'il fût un essayiste, critique et écrivain prolifique, son projet du fantastique réel est toujours resté vague, incohérent, éclaté, ne s'enfermant pas dans une formule unique et évoluant au cours de la longue aventure créatrice de l'auteur. Les deux essais aux titres hautement prometteurs - Poétique des éléments et des mythes (1966) et Le fantastique réel (1967) - s'avèrent passablement décevants pour à celui qui voudrait y trouver un discours construit sur les bases de présupposés intellectuels, logiques ou rationalistes et déterminer la nature de son esthétique. Ces ouvrages constituent un mélange assez disparate de considérations diverses, flottantes et parfois même contradictoires, formulées au cours de sa vie, et qui ne portent que partiellement sur sa conception du fantastique réel.

Il en est de même avec ses fictions. Bien qu'Hellens déclare qu'on peut «grouper l'ensemble de [s]es productions sous la dénomination réalités fantastiques $»^{3}$, la plupart de ses œuvres ont bien peu à faire avec cette esthétique. Il cultive non seulement le roman réaliste (La femme partagée), mais les possibles les plus divers de l'écriture de l'étrange - le fantastique traditionnel qu'il appelle « extérieur » (Nocturnal), le merveilleux à coloration symboliste (Clartés latentes), des romans oniriques surréalistes (Mélusine) ou fantaisistes (Eil-de-Dieu) et, finalement, le fantastique réel qui pourrait désigner seulement quelques textes. Son œuvre constitue un véritable caléidoscope, une mosaïque particulièrement bigarrée d'ouvrages qui prolongent le naturalisme et le symbolisme, annoncent le surréalisme et s'inspirent de diverses esthétiques novatrices, telles que le futurisme, le cubisme et l'art nègre. L'auteur mélange au sein des mêmes recueils, inscrits à l'emblème du fantastique réel, des contes appartenant à ces catégories bien différentes. Deux ouvrages aux titres thématiques connotatifs, ô combien leurrants - Réalités fantastiques (1923) et Nouvelles Réalités fantastiques (1941) - en constituent les exemples les plus probants : hétéroclites esthétiquement, ils ne nous donnent qu'une idée très floue de son concept du fantastique réel ${ }^{4}$.

Ceci ne veut pourtant pas dire que la pensée d'Hellens manque de rigueur et d'incohérence. Loin d'en faut. Son imprécision s'explique par sa conception du fantastique réel. En effet, il ne le considère pas comme un «genre », mais plutôt comme « une façon de voir, de sentir, d'imaginer » (HELlENS, 1991 : 9). Ce n'est pas pour lui une formule, une recette littéraire, mais une attitude en face de la réalité, un instrument d'investigation psychologique et d'expression littéraire. Il refuse de lui donner une définition fixe et universelle, car « on n'atteint pas aux sources ni aux embouchures du fantastique réel par le truchement des mots du vocabulaire »

\footnotetext{
${ }^{3}$ Dans une interview accordée à Guy le Clec'h, in : (DE BoshÈre, MANOLL, Le CleC'H, 1956 : 138).

${ }^{4}$ Il y regroupe, sous une étiquette unique, des textes différents. Voir à ce sujet l'article «Franz Hellens » dans Encyclopédie du fantastique (TRITTER, BRUNEL, 2010 : 394-400). 
(HELlENS, $196: 53$ ), et fustige les fantastiqueurs méthodiques et techniques qu'il compare à des « acrobates sans passion » (HELLENS, 1991:16) :

Mon propos n'est pas d'éducation ; pas davantag $\square \mathrm{d} \square$ critiqu $\square \mathrm{J}$ 'ai de la lecture, comme tout le monde. Peu et réservée. Sans suite préméditée. Incapable de classement, d'ordre chronologique, si ce n'est à vue de nez et selon les besoins. Quelques idées seulement, plus senties que méditées. Impressionniste à ma manière et dans le pointillé. Que le lecteur n'attende pas de moi un enseignement suivi ou un résumé complet. Ce serait au-dessus de mes capacités. Une sorte de tableau, un paysage disparate, brossé à coup de mémoire vécue, mais sans ordre. Des appréciations tranchées, pourtant sincères, et quelques essais d'explication, à mon propre usage, sur tels cas tout personnels (HELLENS, 1991 : 9-10).

Pour éclaircir cette nébuleuse notion et saisir son essence, il nous faut donc survoler d'un regard panoramique l'immense masse que constitue sa production hétéroclite, repérer ses idées sur le fantastique réel, articulées dans ses essais théoriques et illustrées dans ses textes littéraires. Notre travail s'apparentera donc à l'assemblage d'un puzzle, à la recherche des pièces dispersées dans différents textes qui, une fois réunies, composeront, nous l'espérons, une image cohérente.

Mais avant de s'aventurer dans l'œuvre hellensienne, il convient de remonter à la généalogie du fantastique réel, donc à Edmond Picard qui, en 1887, introduit cette notion dans le monde littéraire belge et trace ses premiers contours, avant de l'appliquer à Hellens. En empruntant ce syntagme oxymorique à la critique française, à Émile Deschamps ou à Jules Claretie ${ }^{5}$, Picard la naturalise comme une détermination propre à la production littéraire belge et tend à en faire sa spécificité, un trait définitoire de l'identité culturelle des Belges. Il l'utilise pour la première fois dans un long essai éponyme, publié dans Le Juré (PICARD, 1887 : 27-47), dans lequel il invite ses compatriotes à faire leur deuil du fantastique extérieur des romantiques et à poser un regard nouveau sur la réalité qui, d'après lui, cache le mystère :

Il [le fantastique réel] a sur la vie, sur les hommes, sur les choses des vues défiantes et des réflexions inquiétantes. Tout n'est pas aussi simple qu'on le croit. Les événements n'ont pas la logique que notre pénétration leur prête. Il y a partout des dessous, des mystères. [...] le monde est plein d'étrangeté (PICARD, 1887 : 33-35).

Inspiré par les idées positivistes, PICARD tend à chasser le surnaturel explicite et vante les mérites d'une psychologie de limites, censée écrire « l'intrusion sourde [...] du déséquilibre dans la pensée » (1887: 40). Il propose d'accorder le privilège aux manifestations les plus intérieures de l'étrange, à l'observation des « maladies psychologiques raffinées », tout en renonçant à «l'analyse psychologique » qui n'est que l' «aliment d'un examen froid, scientifique, même dans la littérature la plus novatrice » (PICARD, 1887 : 40). Ensuite, il exhorte à regarder avec minutie le réel pour dénicher le mystère qui se tapit derrière le rideau de l'apparence immédiate

${ }^{5}$ É. Deschamps l'emploie pour l'intitulé de son recueil Réalités fantastiques (1954), J. Claretie y recourt dans la préface aux Histoires incroyables (1885) de Jules Lermina. Voir : (LysøE, 1995 : 85 $101)$. 
et que « les yeux vulgaires [...] ne voient pas » (PICARD, 1887 : 35). Son fantastique s'appuie donc sur la réalité et correspond à un regard, à une vision singulière de l'artiste ; il n'est plus dans l'objet, mais dans l'œil. Pour Picard, la réalité est dans son essence fantastique, il faut seulement savoir la regarder ${ }^{6}$.

En 1910, Picard reprend la formule et les idées, présentées dans la préface du Juré à propos des Hors-le-Vent d'Hellens, dans son article retentissant «Franz Hellens. Le fantastique réel », publié dans La Chronique le 18 décembre. Hellens se les approprie et tâche, sa vie durant, de se singulariser par l'interprétation personnelle qu'il fait de cette catégorie. La lecture ciblée de ses essais et de ses fictions non réalistes permet de formuler le premier constat: le fantastique réel hellensien se fonde essentiellement sur l'harmonieuse conciliation du réel et de l'irréel. Ainsi lisons-nous dans Journal de Frédéric, à la date du 5 février : «le fantastique sort tout droit de l'homme : il n'abandonne jamais la réalité, il l'élargit » (HELlENS, 1935 : 7). Cette fusion inhérente trouve son expression plus nette dans Retour du fantastique :

Ici, la réalité n'est pas transposée, avec tous ses éléments visibles et naturels, sur un plan surnaturel, elle est transformée, incorporée au surnaturel ; il se fait un va-et-vient si rapide et nécessaire, que le lecteur assiste au miracle d'une perpétuelle transsubstantiation (HELLENS, 1992 : 103).

L'insolite ne fait pas irruption dans le réel, ne lui est pas extérieur, mais il en est la partie intégrante. Cet usage du surnaturel différencie le fantastique réel du fantastique : il ne relève pas d'une «intrusion brutale du mystère dans le cadre de la vie réelle » (CASTEX, $1987: 8$ ), ne provoque pas la « rupture des constantes du monde réel »(VAX, $1974: 6) ; 1 \square$ mystèr $\square$ apparti $\square$ nt à l'ordre et aux possibles de ce monde et constitue un élément immanent de la réalité. HELLENS explique : «Le réel n'est pas seulement ce que nos sens physiques perçoivent, il est, déjà dans sa course et ses évolutions, mélangé d'irréel, c'est-à-dire, non pas de métaphysique, mais de physique survolté » (1991 : 71). Le fantastique réel, tel qu'HELLENS le conçoit, est une « réfraction insolite du réel quotidien » $(1991: 13,73)$; il « dépayse la réalité » (1992: 103). La tâche que l'écrivain s'assigne est de faire émerger cette part mystérieuse du réel: "s'avancer tellement dans le frisson du concret que l'imaginaire éclate comme de lui-même de la réalité élue, tel est le cheminement le plus secret mais le plus efficace d'une certaine conception tout irrationnelle de l'esprit et de la sensibilité » (HELLENS, 1991 : 17-18).

Pour Hellens, comme pour Picard, le fantastique est une question d'optique. L'auteur de Mélusine est un observateur visionnaire qui, à force de scruter attentivement la réalité ambiante, familière et prosaïque, parvient à y entrevoir ce qui est celé au regard. Il pense que le réel, à force d'être contemplé, finit par libérer

\footnotetext{
${ }^{6}$ P. Fierens dira, en 1947, la même chose sur l'optique qu'adoptent les peintres flamands pour scruter la réalité : «On regarde la vie, à l'œil nu ou bien à la loupe : à force de la regarder, on la voit changer de visage, se transformer, se déformer, se recomposer sur le plan du songe, de la féerie ou de la fantasmagorie. Rien d'arbitraire, rien de purement gratuit dans l'invention de cet autre monde ». (FIERENS, $1947: 7$ ).
} 
une sorte de mystère et révéler son envers ignoré qui est fantastique. La nouvelle $L a$ courge (1919) l'illustre parfaitement. Elle met en scène un personnage qui, en scrutant attentivement une courge, offerte par un ami et transformée en gourde, y voit aussitôt son passé lointain où il était moine ou ermite hindou, quelque part dans un pays exotique où, sous le soleil, poussaient des hibiscus et des palmiers. Cette découverte le déroute, certes, mais aussi le fascine et le ravit, car il sait maintenant que la mort n'est pas une fin définitive, qu'il y a une autre vie au-delà du réel. Il conclut, serein, au terme du récit :

J'ignore sous quelle forme je reparaîtrai ailleurs, après cette vie. Mais je sais qu'une autre suite d'existences diverses m'attendent encore au sortir de celle-ci. Il est regrettable que tous les hommes qui pensent et s'inquiètent ne reçoivent pas comme moi quelque courge pour leur révéler ce que la réflexion et toute la philosophie du monde ne pourront jamais leur apprendre (HELLENS, $2009: 21)$.

Il en est de même dans Ce lourd silence de pierre où le regard du personnagenarrateur lui fait entrevoir un ailleurs ou, comme le dit joliment Michel Brion, «l'autre face du visible» (BRION, 1957 : 137), lors d'une visite dans un musée de sculpture, situé en plein air dans la banlieue parisienne. Il s'y rend pour voir une statue taillée par son ami de jeunesse, Maurice Bernier, mort il y a trente-cinq ans, qui s'est représenté lui-même de façon peu conventionnelle, c'est-à-dire en toilette de ville : en pardessus, le chapeau sur la tête, l'air et l'attitude d'un promeneur. Il la retrouve et constate, en considérant les traits du visage de l'homme en granit, que celui-ci ne ressemble point à son ami. Il ne reconnaît pas non plus la manière de l'artiste. Néanmoins, à force d' « examiner attentivement » l'ouvrage, il parvient à y voir ce que son regard ne percevait pas au premier regard :

[...] les traits de pierre commencèrent à se découvrir, avec cet air prudent, mais irrésistible, de certains objets qui prennent dans la demi-obscurité du soir leur véritable physionomie, et je ne m'étonnai pas d'avoir pu prononcer presque tout haut le nom de mon ancien ami [...] (HELLENS, $2009: 178)$.

C'est ainsi qu'il finit par voir l'invisible et pénétrer dans une autre réalité, jusqu'alors insoupçonnée. Les événements qui suivent montrent qu'il s'agit d'un arrière-pays de la réalité par excellence fantastique : la statue de pierre ouvre la bouche et lui parle; $\square$ nsuit $\square \square 1 \square$ s'anime pour visiter avec l'homme le musée et, la nuit, se promener à ses côtés à Paris.

Toutefois, le privilège de voir l'autre côté du miroir de la vie n'est pas donné à tous. Pour accéder à ces arrière-plans mystérieux, il faut posséder une perception particulièrement aiguisée et sensible, une « voyance » de qualité, supérieure, qui va bien au-delà du paravent des apparences et démasque les profondeurs secrètes cachées au regard. Cette sensibilité hypertrophiée est, selon l'écrivain, le propre des artistes, ce qui explique la profusion de poètes, peintres, architectes, sculpteurs ou musiciens dans ses narrations. Laissons parler Hellens : « Pour tout homme capable de s'observer, de se sentir, l'existence la plus positive offre, à quelque moment, de ces circonstances vraiment extraordinaires où les sens sont comme retournés, où la 
conscience prend un tour insolite et s'égare dans d'inextricables fantasmes » (HELLENS, 2009 : 161).

Nous pouvons observer, dès lors, que ce regard perçant s'aventure toujours dans la même direction : au-delà de l'horizon quotidien dont les frontières se lézardent et s'ouvrent soudainement sur un univers caché. Voici le véritable siège du surnaturel, dans le fantastique réel, que Thomas Steinmetz appelle «le fantastique de révélation » : la perception des personnages, blasée et lissée par l'habitude, a comme « un sursaut de candeur » et retrouve une sensibilité qu'ils ne lui connaissent pas (2007 : 49). Tout se passe comme s'ils se réveillaient en pleine veille et voyaient ce qui était jusqu'alors invisible, caché sous le couvercle des apparences. L'essence du fantastique réel se trouve donc moins dans l'événement surnaturel que dans l'expérience de la conscience, dans la modification du regard qui se pose sur la réalité ambiante. Thomas Steinmetz note à ce propos :

L'illusion est partout, et le monde tel que nous le pensons est un mensonge, une agréable tromperie qui cache une complexité immense, dont nous avons cent fois pressenti l'existence au cours des moments très intenses, mais immédiatement oubliés, recouverts, difficiles à examiner parce que l'intelligence même, qui ne les connaît pas, est impuissante à les saisir, à en garder la trace dans la mémoire. [...] Soudain, au moment où l'on s'y attend le moins, la conscience comme assoupie s'ouvre à ce qu'elle perçoit immédiatement comme vérité, mais qui lui apparaît de façon diffuse, brève, sans aucune raison apparente : voilà (STEINMETZ, $2007: 50$ ).

Qui plus est, ce fantastique réel, qu'Hellens appelle également « intérieur », se fonde rarement sur une donnée surnaturelle et relève de la réalité de tous les jours, bien familière et prosaïque, dont le spectacle le charme et l'inspire. Nous lisons dans Documents secrets :

Le seul fantastique valable est celui de la vérité la plus absolue. Il suffit de grossir légèrement un objet, une figure, une forme quelconque, pour qu'ils nous apparaissent dans leur réalité féerique, c'est-à-dire différents et pourtant semblables à eux-mêmes ; à la fois plus rapprochés, tt, je ne sais par quelle magie, plus éloignés de nous (HELLENS, 1958 : 272).

L'écrivain exclut le fantastique qui fait appel à Dieu, au diable, aux fantômes et à d'autres entités de l'au-delà. Ses « dieux et démons sont sur terre, nés vivants du sol des choses, du cœur et du cerveau de l'homme » (HELLENS, 1991 : 13). S'il met en scène un phénomène surnaturel, comme la revenance (Le brouillard, La mort est une récompense), le double (Blaise et Monique, L'habit du mort), des aberrations spatiales (La cuisine des fous) ou un objet transitionnel (Le lourd silence de pierre, Un voyant ou Le portrait), celui-ci n'est jamais maléfique, monstrueux ou épouvantable ${ }^{7}$. Il n'est même pas, comme dans le fantastique, un élément primordial mais secondaire, sinon accessoire, dans l'économie du récit. L'essence du fantastique réel hellensien se trouve essentiellement dans une expérience de la

\footnotetext{
${ }^{7} \mathrm{M}$. BRION constate à ce propos : « Ses réalités fantastiques ne sont pas des réalités surnaturelles : il montre l'autre face du visible, il amène à la clarté les régions obscures du cœur et de l'esprit » (1957: 137).
} 
conscience, dans une modification du regard que le personnage, voyeur lucide, porte sur la réalité familière.

Pour Hellens, il suffit de si peu pour que le fantastique jaillisse : d' un glissement, d'une légère entorse à la vision ordinaire des choses. Picard l'a bien compris lorsqu'il a dit à propos des Hors-le-Vent :

Tout va, certes, naturellement du monde. [...] Rien n'est plus simple. Et pourtant... ne va-til pas arriver quelque chose ? Il y a eu un soupir dans le corridor... le vent ? Il y a eu un craquement dans la boiserie, une ondulation dans la grande tapisserie ou s'étale un chimérique passage. Je suis inquiet... je suis ému... Une corde du piano casse et vibre dans sa caisse fermée. [...] Qu'estce ?... C'est étrange, tout cela... Pénétrais-je dans un monde invisible ? Non, c'est le Réel, mais le réel vu, senti en ses accidents énigmatiques, avec intensité (PICARD, $1910: 1$ ).

Hellens déplace le centre de gravité du genre : chez lui, le surnaturel est moins dans les choses mêmes que dans la perception et, ensuite, dans la conscience du personnage. Les événements bouleversants y ont rarement lieu, ils ne sont pas indispensables pour que le sentiment fantastique naisse. Très souvent, il ne se passe rien ou presque, et pourtant le personnage, en portant un regard autre sur l'entourage, aperçoit que quelque chose a changé, sans qu'il y ait eu changement, que la réalité est la même et pourtant autre. Aucune intervention d'un élément extérieur ne survient, c'est l'état d'esprit du protagoniste qui se modifie, voilà la pierre angulaire du fantastique réel hellensien. Francis de Miomandre l'explique parfaitement :

Celui qui contemple avec attention n'importe quel réel ne tarde pas à s'apercevoir qu'il se trouve en face d'une chose extraordinaire, dépassant de tous côtés les bornes de son apparence immédiate, pour plonger à même le mystère. Une fleur qui penche sur sa tige, le cri d'un passant, le regard soudain d'une femme, une bouffée de parfum, en vous arrachant à la distraction qui est, hélas! l'état mental ordinaire des gens, vous fait entrevoir un monde presque illimité concentriquement ébranlé autour de ce fait insignifiant pour les autres (MiOMANDRE, 1957 : 126).

Le fantastique réel ne tient pas aux phénomènes surnaturels, mais à une certaine appréhension du réel. Il se fonde sur un regard plus vrai sur la réalité concrète, regard révélateur l'entrevoyant infiniment plus riche, plus complexe et foisonnante qu'une perception ordinaire, lissée par l'habitude, ne perçoit pas. Les fictions hellensiennes montrent qu'une telle découverte du mystère au cœur du quotidien constitue un mode d'approche et de connaissance du monde : elle déverrouille le carcan du réel, élargit la réalité et dilate le potentiel signifiant de l'existence. En ce sens, le fantastique réel possède, pour lui, une valeur cognitive indéniable.

Cette valeur révélatrice, Hellens l'alloue aussi aux rêves ${ }^{8}$. Il dit et redit que le rêve, éveillé ou non, est le lieu et le passeur de la vérité, "que tout rêveur est un voyant » (HELLENS, 1992 : 73). Selon l'écrivain, seul le rêve donne accès à la vraie vie, permet d'atteindre une vérité supérieure de l'être, de vivre une existence

\footnotetext{
${ }^{8}$ Sur la place et les fonctions du rêve dans la conception du fantastique réel hellensien, voir l'article pertinent de Michel OTTEN, Franz Hellens et le réalisme fantastique (1985 : 41-53).
} 
plénière et de réaliser les plus profonds désirs. Il confère à l'homme un sixième sens, « est une porte ouverte sur l'infini » (HELLENS, 1992 : 73). HELLENS constate : «La réflexion n'évoque que des chimères, le rêve constitue la réalité » (1958:91). Il ajoute en d'autres lieux : "Quoi de plus réel, de plus vrai, de plus détaché des contingences raisonneuses, que le rêve, cauchemar ou songe bienheureux!» (HELLENS, 1991 : 68). De la sorte, il ne faut pas s'étonner qu'il fasse du rêve un générateur du fantastique. En effet, de nombreux contes pivotent autour du motif de la contamination du réel par le rêve : ceux-ci s'y entremêlent et s'y équilibrent à un tel point qu'il est impossible de deviner à quel endroit du récit on glisse d'un monde à l'autre, où finit l'un et où commence l'autre, car, comme le note Auguste GRISAY, «seul, un mince écran imperceptible les sépare » (1962: 31). Le personnage d'Au repos de la santé, ayant rêvé la rencontre nocturne d'une fille en robe écarlate, dans une auberge où le temps s'était arrêté, se demande au réveil s'il a vraiment passé la soirée avec la fille ou s'il a rêvé.

Hellens développe une écriture d'indécision qui donne aux choses un aspect double et fait naître l'hésitation sur laquelle Todorov appuiera sa fameuse définition du fantastique. Le lecteur ressent fortement cette ambiguité car, dans les fictions hellensiennes, rien n'est transgressé dans l'ordre du monde naturel. Les rêves sont vraisemblables, très réels, et de plus, ils ne sont même pas présentés comme rêves, mais comme des épisodes survenant dans un cadre quotidien et relativement banal. Pour les contemplateurs attentifs de la réalité, le monde du rêve, purement subjectif, et le monde de la réalité objective s'entretissent si imperceptiblement et si étroitement que la question de savoir s'ils vivent une aventure onirique ou une aventure réelle semble vaine.

Quant à l'effectivité du fantastique réel, Hellens renonce aux terreurs paniques au profit de sentiments plus feutrés et plus ambivalents. Il le formule clairement :

Le fantastique réel n'est pas nécessairement générateur d'épouvante, mais il n'exclut ni tremblement ni l'angoisse; il n'est pas indispensable de faire appel à des monstres, si beaux soient-ils, infernaux ou terrestres, pour obtenir l'effet de trouble. Considéré dans sa mesure ou sa démesure les plus efficientes, il ne secoue que les nerfs de l'âme et les fibres musicaux [sic !] d'une sensibilité toute spirituelle (HELLENS, $1991: 61$ ).

L'écrivain ne joue pas sur le clavier de l'épouvante et préfère des émotions plus subtiles, plus tempérées, plus intimes, comme un dérangement, une inquiétude ou une angoisse diffuse qui engendrent un effet de trouble et laissent planer un malaise. La lecture de ses contes révèle que l'attitude des personnages envers l'expérience insolite qu'ils vivent s'avère complexe et contradictoire : en même temps qu'ils sont troublés, ébranlés par le spectacle qu'ils voient, ils ressentent un étrange plaisir, une attirance qui les pousse au-devant du phénomène surnaturel. Citons, à cet égard, le narrateur de La cuisine des fous qui, dans une obscure loge de concierge, entrevoit une autre réalité sourdre sous les apparences. C'est là, sans aucun doute, ce qui l'attire : «Moi-même n'avais-je pas respiré l'haleine de ces hôtes, sans que je pusse m'expliquer quelle séduction se dégageait de leur présence ?» (HELLENS, 1912 : 28). Il en est de même avec le personnage-narrateur de La courge qui, dès sa 
première «vision» du passé lointain, rêve de revivre cette bouleversante expérience. Il avoue :

Depuis, je ne passai aucun jour sans éprouver le désir intense de retrouver cette inquiétante hallucination. [...] J'abandonnais sans scrupule mes spéculations quotidiennes pour me jeter à tout moment vers cette énigme qui m'obsédait. [...] Ma curiosité vibrait comme une corde tendue à l'excès, et le désir confina bientôt à la douleur (HELLENS, 2009 : 16).

Bien qu'Hellens se soit toujours complu dans l'art de la divagation, il a réussi à proposer une esthétique qui a séduit et continue à séduire les auteurs belges, ses successeurs. Il reste un des grands inspirateurs du fantastique belge que d'illustres écrivains d'hier et d'aujourd'hui, tels Marcel Thiry, Jean Muno, Thomas Owen tardif, Maurice Câreme ou Bernard Quiriny, ont suivi ou suivent encore dans la recherche du surnaturel dans le réel, non pour les dissocier et opposer, mais pour révéler leur fascinante conciliation. Inspirée de sa vision décalée vis-à-vis du réel, leur imagination les amène à prendre des chemins de traverse qui distraient du traintrain de tous les jours, éclairent autrement la réalité et conduisent vers des ailleurs fantastiques. C'est en ce sens que Jean-Baptiste BARONIAN affirme que l'œuvre fantastique constitue « une fascinante école du regard », une façon particulière de voir la réalité (1977: 97).

\section{BIBLIOGRAPHIE}

BARONIAN Jean-Baptiste (1977), Un nouveau fantastique, Lausanne, L'Âge d'Homme.

Boshere Jean de ; MANOLl Michel ; Le CleC'H Guy (1956), Franz Hellens, Lyon, Armand Henneuse.

BRION Michel (1957), «Le fantastique dans l'œuvre de Franz Hellens », in : Hommages à Franz Hellens, Paris, Albin Michel, p. 131-139.

DECKER Jacques de (1990), «Jean Muno», in: Les années critiques. Les Septentrionaux, Bruxelles, Ercée, p. 4051.

DENIS Benoît; GRAVEz Damien (2002), Textyles (Du fantastique réel au réalisme magique), $\mathrm{n}^{\circ} 21$.

FIERENS Paul (1947), Le Fantastique dans l'Art flamand, Bruxelles, Éd. du Cercle d'Art.

GRISAY Auguste (1962), L'œuvre de Franz Hellens, Liège, Éd. de l'Essai.

HALEN Pierre (1985), «Un certain regard sur le monde. Le fantastique réel dans l'œuvre de Franz Hellens », in : OTTEN Michel (éd.), Écritures de l'imaginaire, Bruxelles, Labor, p. 45-67.

Hellens Franz (1958), Documents secrets (1905-1959). Histoire sentimentale de mes livres et de quelques amitiés, Paris, Albin Michel.

HELLENS Franz (1991), Le fantastique réel, Bruxelles, Labor, [1967].

Hellens Franz (1992), Retour du fantastique, in : "Style et Caractère », in : Un balcon sur l'Europe, textes établis et présentés par GORCEIX Paul, Bruxelles, Labor, coll. «Archives du futur ».

HeLlens Franz (2009), Le double et autres contes fantastiques, Bruxelles, Luc Pire, coll. « Espace Nord ». 
LYsøE Éric (1995), «Franz Hellens et le fantastique réel : Quelques jalons pour l'arpenteur », in : La littérature belge de langue française. Itinéraires et contacts de cultures, $\mathrm{n}^{\circ}$ 20, Paris, L'Hartamman, p. 85-101.

MiomANDRE Francis (1957), «Pensées sur Franz Hellens », in : Hommages à Franz Hellens, Paris, Albin Michel, p. 125-127.

OTTEN Michel (1985), «Franz Hellens et le réalisme fantastique», in: Le fantastique dans les lettres françaises de Belgique. Cahiers du CERCLEF, $\mathrm{n}^{\circ} 3$, Université de Paris XII, p. 41-53.

PICARD Edmond (1910), «Franz Hellens. Le fantastique réel », La Chronique, 18 décembre 1910, p. 1-3.

PICARD Edmond (1889), Le Juré, Bruxelles, Vve Monnom.

SiweK Ryszard (2001), Od De Costera do Vaesa. Pisarze belgijscy wobec niezwyktości, Kraków, Wyd. Naukowe Akademii Pedagogicznej.

STEINMETZ Thomas (2007), «Les angles saillants de la conscience : le fantastique de révélation », Revue de littérature comparée, $\mathrm{n}^{\circ} 321$, p. 43-57. 